\title{
Patient Perception of Negative Noninvasive Prenatal Testing Results
}

A. Theresa Wittman, MS, $\mathrm{CGC}^{1,2} \quad$ S. Shahrukh Hashmi, MD, MPH, PhD ${ }^{1,3}$ Hector Mendez-Figueroa, MD 4 Salma Nassef, MS, CGC ${ }^{2}$ Blair Stevens, MS, CGC $C^{1,4}$ Claire N. Singletary, MS, CGC $1,3,4$

${ }^{1}$ Genetic Counseling Program, University of Texas Graduate School of Biomedical Sciences at Houston, Houston, Texas

2 Department of Human and Molecular Genetics, Baylor College of Medicine, Houston, Texas

${ }^{3}$ Department of Pediatrics, McGovern Medical School at UT Health, Houston, Texas

${ }^{4}$ Department of Obstetrics, Gynecology and Reproductive Sciences, McGovern Medical School at UT Health, Houston, Texas
Address for correspondence A. Theresa Wittman, MS, CGC, Department of Human and Molecular Genetics, Clinic Program, Baylor College of Medicine, The Methodist Hospital, Smith Tower, 6565 Fannin Street Suite 901, Houston, TX 77030 (e-mail: wittman@bcm.edu).

Am J Perinatol Rep 2016;6:e391-e406.

\begin{abstract}
Keywords

- noninvasive prenatal testing

- patient perception of negative screening

- negative noninvasive prenatal testing

- prenatal screening for aneuploidy

- limitations of prenatal screening

- prenatal screening

- genetic counseling

Objective To determine patient perception of residual risk after receiving a negative non-invasive prenatal testing result.

Introduction Recent technological advances have yielded a new method of prenatal screening, non-invasive prenatal testing (NIPT), which uses cell-free fetal DNA from the mother's blood to assess for aneuploidy. NIPT has much higher detection rates and positive predictive values than previous methods however, NIPT is not diagnostic. Past studies have demonstrated that patients may underestimate the limitations of prenatal screening; however, patient perception of NIPT has not yet been assessed.

Methods and Materials We conducted a prospective cohort study to assess patient understanding of the residual risk for aneuploidy after receiving a negative NIPT result. Ninety-four participants who had prenatal genetic counseling and a subsequent negative NIPT were surveyed.

Results There was a significant decline in general level of worry after a negative NIPT result ( $p=<0.0001)$. The majority of participants $(61 \%)$ understood the residual risk post NIPT. Individuals with at least four years of college education were more likely to understand that NIPT does not eliminate the chance of trisomy $13 / 18(p=0.012)$ and sex chromosome abnormality $(p=0.039)$, and were more likely to understand which conditions NIPT tests for $(p=0.021)$, compared to those women with less formal education.

Conclusion These data demonstrate that despite the relatively recent implementation of NIPT into obstetric practice, the majority of women are aware of its limitations after receiving genetic counseling. However, clinicians may need to consider alternative ways to communicate the limitations of NIPT to those women with less formal education to ensure understanding.
\end{abstract}

DOI http://dx.doi.org/

$10.1055 / \mathrm{s}-0036-1594243$. ISSN 2157-6998.
Copyright $\odot 2016$ by Thieme Medical Publishers, Inc., 333 Seventh Avenue, New York, NY 10001, USA. Tel: +1(212) 584-4662.
License terms

(c) (1) $\ominus$ (\$) 


\section{Background}

Chromosomal aneuploidy is estimated to occur in $1 / 160$ live births, the vast majority consisting of trisomy 21 , trisomy 18 , trisomy 13 , and sex chromosome conditions. ${ }^{1}$ Before the advent of recent prenatal testing options, women seeking information about aneuploidy in their pregnancy generally had two options: (1) invasive diagnostic testing that confers a risk for miscarriage or (2) noninvasive screening, which generally had false-positive rates of $5 \%$ or more and positive predictive values (PPVs) between 1 and $10 \%$.,3

In November 2011, noninvasive prenatal testing (NIPT), or prenatal cell-free fetal DNA screening, became clinically available for use in high-risk populations. NIPT was validated in a high-risk population in multiple studies, all of which have shown similar accuracies for aneuploidy detection. ${ }^{4-7}$ The most recent metaanalysis by Gil et al in 2015 analyzed data from 37 relevant studies and determined that NIPT detection rates for the most common aneuploidies are approximately $99.2 \%$ for trisomy 21 , $96.3 \%$ for trisomy $18,91 \%$ for trisomy 13 , and 90 to $93 \%$ for sex chromosome aneuploidy. ${ }^{8}$ While the detection rates and PPVs for NIPT are increased in comparison to other methods of prenatal screening, NIPT is not a diagnostic test, and a negative NIPT result does not guarantee a pregnancy is unaffected. ${ }^{9}$ NIPT laboratories' marketing efforts and Web site content often focus on the detection rate rather than PPV or residual risk. ${ }^{10}$ It is unclear whether the general patient population understands this distinction, which may have implications for downstream uptake of invasive testing and emotional preparation at birth. $^{11,12}$ Therefore, we conducted a cross-sectional study to assess patient understanding of the residual risk for trisomy 21 , trisomy 18 , trisomy 13 , and sex chromosome aneuploidy after receiving a negative NIPT result.

\section{Methods}

From August 1, 2015, through January 29, 2016, women who were at least 18 years old, English or Spanish speaking, and had been consented for NIPT during their genetic counseling appointment were invited to participate in the study. Only those women who had formal genetic counseling with a prenatal genetic counselor were recruited and consented. Participating centers were staffed by University of Texas Health and Baylor College of Medicine prenatal genetic counselors in the Houston, Texas, area and approved by the institutional review boards at the University of Texas Health and Memorial Hermann Hospital (HSC-MS-15-0444), Baylor College of Medicine and affiliated Texas Children's Hospital (H-37683), and the Harris Health System (15-09-1193). Those patients willing to take part signed a consent form agreeing to be contacted after their NIPT results were available (-Appendix A), and only those with a negative result were contacted to participate. The recruited participants were given their NIPT results over the phone by a prenatal genetic counselor. It is standard protocol among the genetic counselors involved in the study to emphasize the limitations of NIPT during the consent process as well as at the time of the results disclosure, including that it is not diagnostic, there remains a residual risk, and it does not test for every genetic disorder. Physical copies of NIPT results were only given upon patient request.

The survey given to those participants with a negative NIPT result consisted of a section designed to assess patient understanding of the limitations of NIPT, a section to assess worry level for various conditions, a section regarding subsequent testing, and a section with demographic information (-Appendix B). An online survey tool, Redcap, was used to securely administer the survey via email and collect the data. Those participants unable to complete the survey via email were called and given the survey over the telephone. Data from telephone calls were manually added to the Redcap dataset. Data were analyzed using STATA, (v.14.1, College Station, TX). Comparison of data between groups was evaluated using chisquare analysis, Fisher exact test, Wilcoxon signed-rank test, or Mann-Whitney test where appropriate. Statistical significance was assumed at a Type I error rate of $5 \%$.

\section{Results}

A total of 231 women agreed to be contacted for the survey. Six women were excluded due to either a positive NIPT result $(n=3)$ or failure to follow-through with the blood draw $(n=3)$. In total, 225 women were contacted after their negative NIPT result and asked to participate in the survey either through email or phone call. Twenty-nine women (13\%) declined to participate after being contacted and 102 women (45\%) were never successfully contacted, leaving a total of 94 participants (42\%) from the original 225 consented. Twelve (13\%) of the surveys were incomplete, the majority of which were missing the last several questions of the survey (-Fig. 1).

The majority of participants ( $59 \%, n=55$ ) were referred to genetic counseling due to advanced maternal age and most identified as non-Hispanic white $(36 \%, n=34)$ or Hispanic (29\%, $n=27)$. The majority of participants $(64 \%, n=60)$ reported having at least a 4 -year college degree ( $\mathbf{- T a b l e ~} \mathbf{1}$ ).

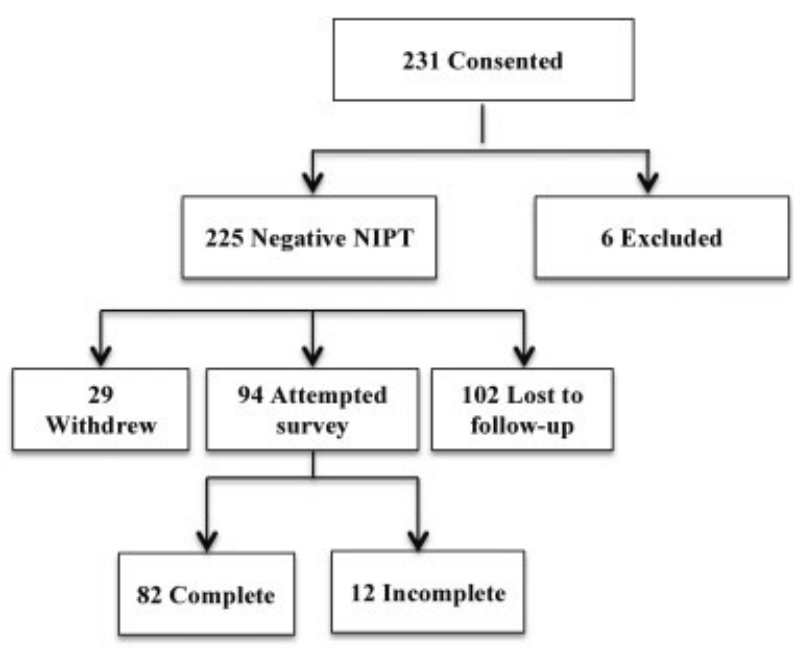

Abbreviations: NIPT, non-invasive prenatal testing

Fig. 1 Survey completion flow diagram. NIPT, noninvasive prenatal testing. 
Table 1 Participant demographics, $n=94$

\begin{tabular}{|c|c|c|}
\hline & $n$ & $\%$ \\
\hline \multicolumn{3}{|l|}{ Ethnicity } \\
\hline Non-Hispanic white & 34 & 36 \\
\hline Hispanic & 27 & 29 \\
\hline African American & 16 & 17 \\
\hline Asian & 11 & 12 \\
\hline Other & 5 & 5 \\
\hline No Answer & 1 & 1 \\
\hline \multicolumn{3}{|l|}{ Household income } \\
\hline Less than $\$ 25,000$ & 10 & 11 \\
\hline$\$ 25,000$ to $\$ 49,999$ & 15 & 16 \\
\hline$\$ 50,000$ to $\$ 74,999$ & 16 & 17 \\
\hline$\$ 75,000$ to $\$ 99,999$ & 15 & 16 \\
\hline$\$ 100,000$ to $\$ 149,999$ & 20 & 21 \\
\hline$\$ 150,000$ or more & 12 & 13 \\
\hline Do not wish to answer & 6 & 6 \\
\hline \multicolumn{3}{|l|}{ Education } \\
\hline Some high school & 1 & 1 \\
\hline High school/GED & 11 & 12 \\
\hline Some college & 22 & 23 \\
\hline 4-year degree & 32 & 34 \\
\hline Graduate degree & 28 & 30 \\
\hline \multicolumn{3}{|l|}{ Marital status } \\
\hline Married/living with partner & 84 & 89 \\
\hline Unmarried & 9 & 11 \\
\hline Do not wish to answer & 1 & 1 \\
\hline \multicolumn{3}{|l|}{ Age (y) } \\
\hline $21-29$ & 15 & 16 \\
\hline $30-34$ & 15 & 16 \\
\hline $35-39$ & 54 & 57 \\
\hline $40-43$ & 10 & 11 \\
\hline \multicolumn{3}{|l|}{ Indication } \\
\hline Advanced maternal age & 55 & 59 \\
\hline Positive serum screen & 11 & 12 \\
\hline Ultrasound abnormality & 11 & 12 \\
\hline Low risk & 9 & 10 \\
\hline Two or more indications & 8 & 9 \\
\hline
\end{tabular}

\section{Patient Perception of Residual Risk Post Negative NIPT Results}

The majority of participants indicated their risk for aneuploidy was decreased but not eliminated post NIPT. A total of $61 \%$ ( $n=57$ ) of women indicated their risk to have a baby with Down syndrome was much lower, $55 \%(n=52)$ indicated that their risk was much lower for trisomy $13 / 18$, and $49 \%(n=46)$ said that their risk to have a baby with a sex chromosome aneuploidy was much lower. A proportion of women also indicated that there was no residual risk after a negative NIPT. Specifically, 34 to $39 \%$ of participants indicated there was no longer a chance for their baby to have Down syndrome, trisomy $13 / 18$, or a sex chromosome aneuploidy after receiving a negative NIPT result. Additionally, participants were asked to indicate their risk to have a baby with a genetic condition other than Down syndrome, trisomy $13 / 18$, or sex chromosome aneuploidy after receiving a negative NIPT result. A total of $13 \%(n=12)$ correctly answered that their risk was not lower than before, $29 \%$ ( $n=27$ ) indicated that there was no longer any chance for their baby to have any genetic problem, $49 \%(n=46)$ answered that it was much lower than before, and $9 \%(n=8)$ responded that it was somewhat lower than before. Women with less than a 4-year college education were significantly more likely to incorrectly respond that there was no longer a risk for their baby to have trisomy $13 / 18(p=0.012)$ or a sex chromosome abnormality ( $p=0.039$ ). Participants with less than a 4-year education also appeared to be more likely to indicate that there was no longer a chance for their baby to have Down syndrome; however, this did not reach significance $(p=0.086)$. Other demographic categories did not show a significant influence on patient perception of negative NIPT results (-Table 2 ). This analysis was performed using Fisher exact test via STATA v. 14.1.

\section{Most Important and Least Important Reasons for Pursuing NIPT}

Participants were asked to share the most important and least important reasons for pursuing NIPT on a scale of 1 to 6 , with 1 being the most important and 6 being the least important. There was no significant difference in how participants ranked their reasons for pursuing NIPT between the various demographic categories (ethnicity, $p=0.586$; income, $p=0.747$; education, $p=0.212$; age, $p=0.373$; indication, $p=0.123$ ) (-Fig. 2). This analysis was performed using Fisher exact test via STATA v. 14.1.

\section{Patient Perception of Conditions Tested by NIPT}

Participants were asked to indicate whether NIPT could test for the following: intellectual disability, autism, diabetes, spina bifida, cleft lip, gender, and structure of the heart. The vast majority of participants $(92 \%, n=86)$ were able to correctly identify that NIPT can test for gender. When looking at the remaining six items from this question, a participant had to indicate that NIPT did not test for the item to be scored as correct. Cleft lip, structure of heart, and spina bifida were considered structural abnormalities, while intellectual disability, autism, and diabetes were considered nonstructural. Those with less formal education were significantly less likely to answer correctly and had lower scores overall $(p=0.021)$. A total of $14 \%$ (5/36) of women with less formal education correctly answered all of the questions in comparison to $37 \%$ $(22 / 60)$ of those women with at least 4 years of college. Overall, the participants were more likely to believe that NIPT could test for structural abnormalities (cleft lip, spina bifida, and structure of heart) versus nonstructural abnormalities (intellectual disability, autism, and diabetes) $(p<0.0005)$ and women with less than a 4-year degree were even more likely than those with higher education to believe that that NIPT 
e394 Patient Perception of Negative Noninvasive Prenatal Testing Results Wittman et al.

Table 2 Patient perception of risk post negative NIPT, $n=94$

\begin{tabular}{|c|c|c|c|c|}
\hline & Down syndrome (\%) & T13/T18 (\%) & $\begin{array}{l}\text { Sex chromosome } \\
\text { aneuploidy (\%) }\end{array}$ & $\begin{array}{l}\text { Any other genetic } \\
\text { condition }(\%)\end{array}$ \\
\hline \multicolumn{5}{|c|}{ Perception of residual risk post negative NIPT } \\
\hline Not lower than before & 0 & 1 & 5 & 13 \\
\hline Somewhat lower than before & 5 & 10 & 7 & 10 \\
\hline Much lower than before & 61 & 55 & 49 & 49 \\
\hline No longer a chance & 34 & 34 & 39 & 29 \\
\hline \multicolumn{5}{|c|}{ Demographic factors and risk perception post negative NIPT } \\
\hline Ethnicity $^{\mathrm{a}}$ & $p=0.440$ & $p=0.119$ & $p=0.177$ & $p=0.130$ \\
\hline Income $^{b}$ & $p=0.588$ & $p=0.540$ & $p=0.166$ & $p=0.752$ \\
\hline Education $^{c}$ & $p=0.086$ & $p=0.012$ & $p=0.039$ & $p=0.159$ \\
\hline $\mathrm{Age}^{\mathrm{d}}$ & $p=0.649$ & $p=0.550$ & $p=0.486$ & $p=0.885$ \\
\hline Indication $^{\mathrm{e}}$ & $p=0.238$ & $p=0.082$ & $p=0.324$ & $p=0.700$ \\
\hline
\end{tabular}

Abbreviations: NIPT, noninvasive prenatal testing; T18/T13, trisomy 13, trisomy 18.

aEthnicity: White Non-Hispanic, Hispanic, African American, Asian/Pacific Islander, Native American, Other, do not wish to answer.

bIncome: $<\$ 25,000, \$ 25,000-\$ 49,999, \$ 50,000-74,999, \$ 75,000-\$ 99,999, \$ 100,000-\$ 149,999, \$ 150,000+$, do not wish to answer.

'Education: Some high school, high school graduate/GED, some college, 4-year degree, graduate degree.

'Age: 21-29, 30-34, 35-39, 40-43.

e Indication: Advanced maternal age, positive serum screen, ultrasound abnormality, low risk, two or more indications.

could test for both structural abnormalities $(p=0.024)$ and nonstructural abnormalities $(p=0.010)$ ( - Fig. 3 ).

\section{Worry Levels before and after Negative NIPT}

Participants were asked to rank their general worry level about having a child with any health problem before undergoing NIPT and then their specific worry levels for Down syndrome, trisomy $13 / 18$, and sex chromosome abnormality after testing via NIPT on a scale of 1 to 5 , with 1 being unconcerned and 5 being very concerned. Similarly, women were asked what their level of concern was to have a baby with any genetic condition after a negative NIPT result. There was a significant decline when comparing the general level of worry before NIPT to each of the worry levels for Down syndrome $(p<0.0001)$, trisomy13/18 $(p<0.0001)$, sex chromosome aneuploidy ( $p<0.0001$ ), and any other genetic condition after a negative NIPT result $(p<0.0001)$. Despite the fact that NIPT cannot reduce risk for all genetic conditions, the majority of participants ( $n=67,70 \%$ ) reported a decrease in worry to have a baby with any genetic disorder (-Fig. 4).

\section{Discussion}

This study aimed to assess patient perception of the residual risk for Down syndrome, aneuploidy other than Down syndrome, birth defects, and other genetic conditions, after a negative NIPT. To our knowledge, this is the first study to examine patient understanding of the limitations of NIPT. The cohort consisted of women who received formal genetic counseling from a prenatal genetic counselor and thus excluded women who received NIPT directly through their obstetrician. The data demonstrate that despite the relatively recent implementation of NIPT into obstetric practice, the majority of women who receive formal genetic counseling by genetic counselors are aware of its

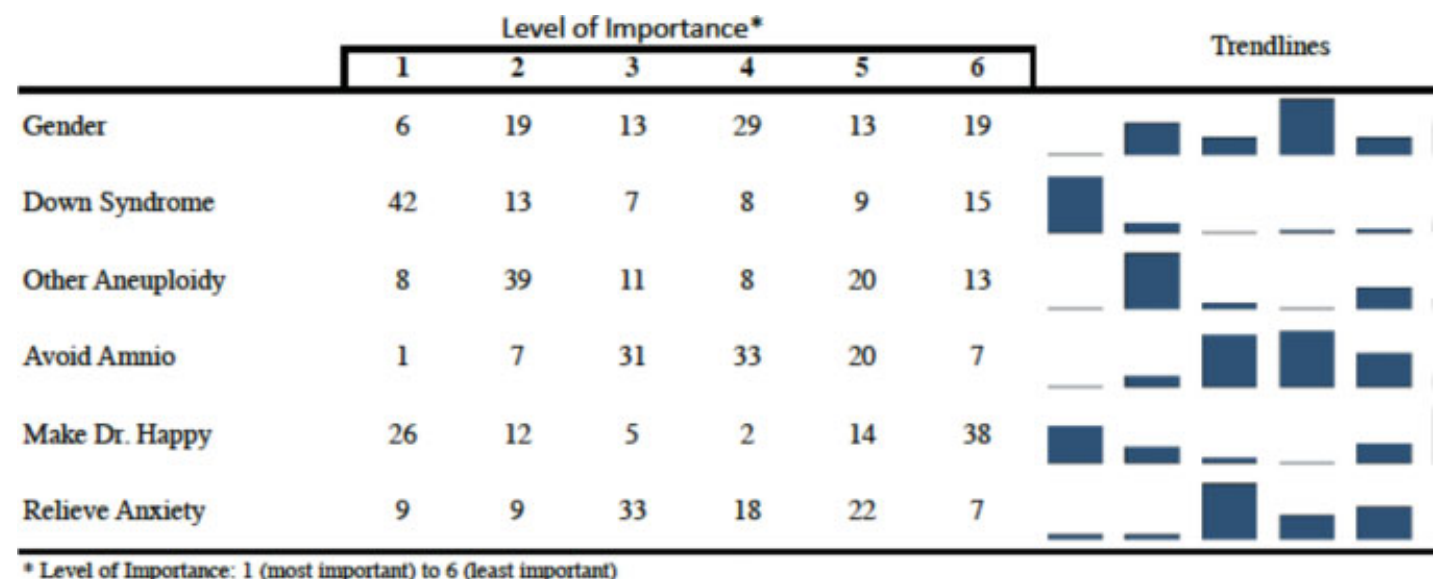

Fig. 2 Most and least important reasons for pursuing NIPT (presented as percentages, $n=85$ ). 


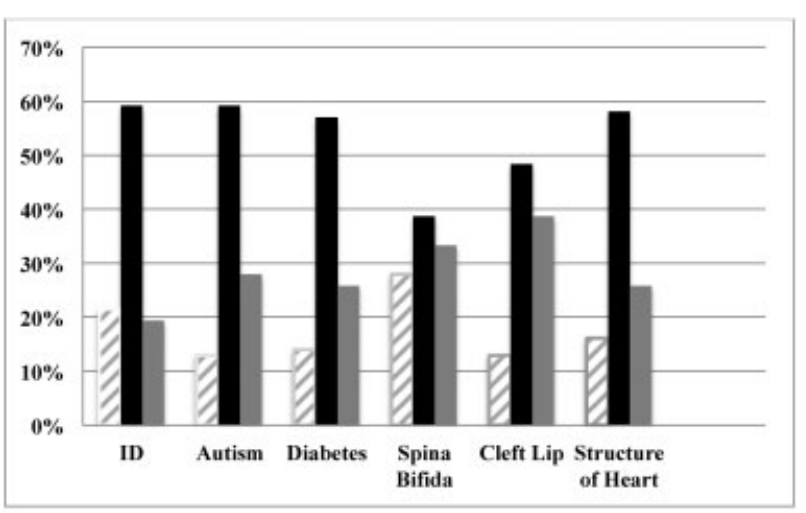

Abbreviations: NIPT, non-invasive prenatal testing: ID, intellectual disability

Fig. 3 Patient perception of conditions tested by NIPT, $n=93$. ID, intellectual disability; NIPT, noninvasive prenatal testing.

limitations. Overall, most participants were able to recognize that NIPT is a screening test and that it significantly reduces the risk for those conditions it tests for, but does not eliminate the risk entirely. Of note for practitioners, patient comprehension of NIPT's screening ability increased significantly with education level. Therefore, practitioners may need to spend additional time discussing the implications of NIPT with patients who have less formal education. Additionally, it should be noted that the genetic counselors involved in the recruitment of this study generally spent 45 to 60 minutes with patients, significantly more time than most obstetric appointments, thus allowing time for discussion and clarification of the limitations of NIPT.

Similarly, many women correctly recognized that NIPT does not test for nonstructural abnormalities such as autism, intellectual disability, and diabetes or structural abnormalities such as heart defects, cleft lip, and spina bifida. Interestingly, participants were more likely to incorrectly respond that NIPT could evaluate for structural abnormalities compared with the nonstructural abnormalities. It is unclear why patient comprehension differed by abnormality. Heart defects and cleft lip are often associated with aneuploidy; therefore, women may have falsely assumed that a negative NIPT reduced the risk for nonaneuploidy-associated heart defects and clefting. In addition, many women at our participating centers had an ultrasound following their genetic counseling appointment. Thus, they may have confused reassurance for structural conditions from the ultrasound with reassurance from NIPT. Furthermore, blood may be drawn to assess alpha fetal protein levels and spina bifida risk at the same time as blood is drawn for NIPT; thus, women may have falsely believed these tests are one in the same. Additional studies may wish to delve into the underlying reasons behind this misunderstanding.

This study also demonstrated that negative NIPT results significantly decreased worry levels of patients regarding having a baby with Down syndrome $(p<0.00001)$, trisomy $13 / 18$ $(p<0.00001)$, and sex chromosome aneuploidy $(p<0.00001)$. This asserts the clinical utility of NIPT to provide appropriate reassurance for women who experience anxiety regarding their risk to have a baby with aneuploidy. However, this study also showed that women who undergo NIPT are also more likely to experience a false decrease in worry levels for conditions not screened by NIPT, suggesting that negative NIPT results may provide patients with false general reassurance in addition to appropriate reassurance for aneuploidy. It is unclear whether or not this is due to lack of understanding related to NIPT or general unfamiliarity with other genetic conditions.

Although the majority of women are likely to understand the limitations of NIPT after genetic counseling, it is clear that education level plays a role in comprehension. Women who had less than a four-year college education were more likely to believe that their NIPT could eliminate their risk to have a child with aneuploidy. Similarly, women with more education were more likely to understand what conditions were included in NIPT. These data are consistent with findings regarding traditional prenatal screening tests. Wong et al. in 2012 demonstrated that women with less formal education were more likely to perceive second trimester ultrasound as more sensitive and diagnostic. ${ }^{13}$ Moreover, past studies of maternal serum screening demonstrated that low health literacy and poor comprehension of the limitations of screening are associated with less years of formal education. ${ }^{14,15}$ Women with low health literacy may also have difficulty with numeracy, confounding their interpretation of the sensitivity, specificity, and PPVs of prenatal screening methods. In 2004, Gates et al examined the role of numeracy on patient understanding and concluded that women with lower levels of literacy and

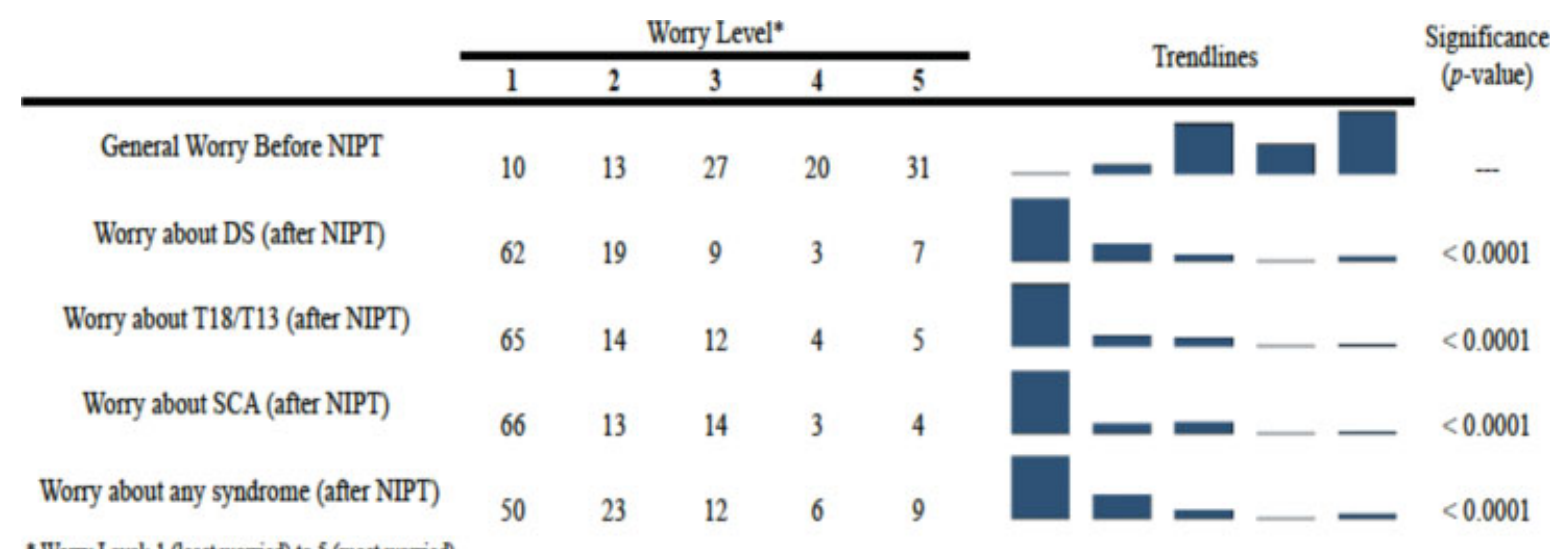

Fig. 4 Worry levels before and after negative NIPT (\%), $n=94$. DS, Down syndrome; NIPT, noninvasive prenatal testing; SCA, sex chromosome aneuploidy; T18/T13, trisomy 13, trisomy 18. 
numeracy have the most difficulty in accurately interpreting information about risk. ${ }^{16}$ The effect of education and health literacy on patient understanding of prenatal screening is an important consideration, as approximately 40\% of women 25 years and older in the United States do not have any formal education beyond high school. ${ }^{17}$

An additional issue that may confuse patients is the manner in which the $99 \%$ detection rate for NIPT is often highlighted by the media and laboratory testing materials rather than focusing on the individual patient's PPV and negative predictive value. Without sufficient background knowledge, women may get the impression that the PPV and detection rates are both 99\%. A study by Mercer et al in 2014 examined the impact of the availability and use of the Internet for gathering information about NIPT. ${ }^{10}$ Their study showed a lack of comprehensiveness and quality of information regarding NIPT obtained through Internet sources. Moreover, many of the Web sites either failed to mention or downplayed information about the limitations and disadvantages of NIPT while simultaneously promoting the accuracy of the test without mentioning the importance of negative predictive value and PPV calculations. It is no wonder that women who research NIPT on the Internet may not appreciate the residual risk for aneuploidy, especially women without advanced formal education.

\section{Conclusion and Future Directions}

The incorporation of NIPT into obstetric practice has proved both exciting and overwhelming. Although it is clear that this new screening option can provide tremendous benefits to women worried about having a baby with a common aneuploidy, proper pretest genetic counseling is essential to ensure that patients are informed of the limitations and potential results from NIPT. This study demonstrated that NIPT invokes similar issues to previous prenatal screening modalities and that providers should be cognizant of the tendency for women with less formal education to overinflate the power of screening to decrease or eliminate their risk for a baby with a genetic condition. Genetic counselors and obstetricians must prioritize communicating information regarding NIPT accurately and clearly, so that women considering it as a screening option may be adequately informed. When possible, attention should be paid to a patient's education level and information should be tailored accordingly. The development of patient-friendly decision aids that clearly state the limitations of screening and what a negative test means may assist in residual risk communication, informed consent, and decision making. ${ }^{18}$

As this was a pilot study with a limited number of participants, more research is needed to examine patient perception of the limitations of NIPT and how this may vary based on patient demographic and geographic factors. Future studies may wish to examine whether the implementation of targeted educational materials and decision aids augment patient understanding of NIPT, especially as the testing platforms are expanded. Furthermore, research should be done to assess patient perception of positive NIPT results and whether or not women who screen positive accurately understand the implications and limitations of the results.

\section{Limitations}

This study was limited by the small sample size. The majority of women were referred for genetic counseling due to either advanced maternal age or positive serum screen. Therefore, we cannot confidently extrapolate to the low-risk population. In addition, $64 \%$ of participants had at least a 4-year college degree. Given the association of education level with understanding, a larger sample size might have allowed for parsing out subgroups from women who had less than a 4- year degree into those with some college, those with a high school diploma, and those without a high school diploma to further stratify the finding. Additionally, the survey used was carefully developed to evaluate the aims of this study; however, this assessment tool has not been validated in other studies. Finally, this research was limited to the greater Houston, Texas, area; thus, these results may not be generalizable to other geographical regions.

\section{References}

1 Driscoll DA, Gross S. Clinical practice. Prenatal screening for aneuploidy. N Engl J Med 2009;360(24):2556-2562

2 Wapner R, Thom E, Simpson JL, et al; First Trimester Maternal Serum Biochemistry and Fetal Nuchal Translucency Screening (BUN) Study Group. First-trimester screening for trisomies 21 and 18. N Engl J Med 2003;349(15):1405-1413

3 Positive predictive value again. BJOG 1999;106(9):vii-viii

4 Palomaki GE, Kloza EM, Lambert-Messerlian GM, et al. DNA sequencing of maternal plasma to detect Down syndrome: an international clinical validation study. Genet Med 2011;13(11):913-920

5 Palomaki GE, Deciu C, Kloza EM, et al. DNA sequencing of maternal plasma reliably identifies trisomy 18 and trisomy 13 as well as Down syndrome: an international collaborative study. Genet Med 2012;14(3):296-305

6 Bianchi DW, Platt LD, Goldberg JD, Abuhamad AZ, Sehnert AJ, Rava RP; MatErnal BLood IS Source to Accurately diagnose fetal aneuploidy (MELISSA) Study Group. Genome-wide fetal aneuploidy detection by maternal plasma DNA sequencing. Obstet Gynecol 2012;119(5):890-901

7 Gil MM, Quezada MS, Bregant B, Ferraro M, Nicolaides KH. Implementation of maternal blood cell-free DNA testing in early screening for aneuploidies. Ultrasound Obstet Gynecol 2013;42(1):34-40

8 Gil MM, Quezada MS, Revello R, Akolekar R, Nicolaides KH. Analysis of cell-free DNA in maternal blood in screening for fetal aneuploidies: updated meta-analysis. Ultrasound Obstet Gynecol 2015;45(3):249-266

9 Neufeld-Kaiser WA, Cheng EY, Liu YJ. Positive predictive value of non-invasive prenatal screening for fetal chromosome disorders using cell-free DNA in maternal serum: independent clinical experience of a tertiary referral center. BMC Med 2015;13:129

10 Mercer MB, Agatisa PK, Farrell RM. What patients are reading about noninvasive prenatal testing: an evaluation of Internet content and implications for patient-centered care. Prenat Diagn 2014;34(10):986-993

11 Tiller GE, Kershberg HB, Goff J, Coffeen C, Liao W, Sehnert AJ. Women's views and the impact of noninvasive prenatal testing on procedures in a managed care setting. Prenat Diagn 2015;35(5):428-433

12 Hall S, Bobrow M, Marteau TM. Psychological consequences for parents of false negative results on prenatal screening for Down's syndrome: retrospective interview study. BMJ 2000;320(7232): 407-412

13 Wong AE, Collingham JP, Koszut SP, Grobman WA. Maternal factors associated with misperceptions of the second-trimester sonogram. Prenat Diagn 2012;32(11):1029-1034 
14 Goel V, Glazier R, Holzapfel S, Pugh P, Summers A. Evaluating patient's knowledge of maternal serum screening. Prenat Diagn $1996 ; 16(5): 425-430$

15 Cho RN, Plunkett BA, Wolf MS, Simon CE, Grobman WA. Health literacy and patient understanding of screening tests for aneuploidy and neural tube defects. Prenat Diagn 2007;27(5): 463-467
16 Gates EA. Communicating risk in prenatal genetic testing. J Midwifery Womens Health 2004;49(3):220-227

17 United States Census Bureau. Educational Attainment in the United States. 2014. Available at: http://www.census.gov/hhes/ socdemo/education/data. Accessed February 18, 2016

18 Vlemmix F, Warendorf JK, Rosman AN, et al. Decision aids to improve informed decision-making in pregnancy care: a systematic review. BJOG 2013;120(3):257-266

\title{
Appendix A
}

\author{
Patient Consent Form
}

\section{INFORMED CONSENT FORM TO TAKE PART IN RESEARCH}

Title: Patient perception of residual risk post negative NIPT results

Letter of Information

HSC-MS-15-0444

\section{Primary Investigator: Claire Singletary}

You are invited to take part in a research study called, "Patient perception of residual risk post negative NIPT results," conducted by Claire Singletary of the University of Texas

Health Science Center at Houston. For this research project, she will be called the

Principal Investigator or PI.

The purpose of this study is to evaluate patient perception of their negative NIPT results. If you decide to take part in the study, the total time commitment is 15 minutes. You are invited to take part in this study because you have elected to proceed with noninvasive prenatal testing (NIPT). Women who choose to participate will be contacted after they receive their NIPT results. You can refuse to answer any questions asked or written on any forms. Participation in this study is voluntary. A decision not to take part in this study will not change the services you receive through the University of Texas Health Science Center at Houston.

If you agree to take part in this survey, you will agree to a 15-minute survey. 
You may not receive any benefit from taking part in this study. The information you provide will help to determine patient understanding of noninvasive prenatal testing. There are no known risks to take part in this study. The only possible risk may be breach of confidentiality. This information collected in the survey responses will not contain identifying information and will be kept on a secure server. You have the alternative to choose to not take part in this study and can withdraw at any time.

There is no cost and you will not be paid to take part in this study. However, upon completion of the survey you can choose to be entered in a drawing to win a $\$ 50$ Target gift card. You will not be personally identified in any reports or publications that may result from this study. Any personal information about you that is gathered during this study will remain confidential to every extent of the law.

If you have any questions about this project please contact study coordinator Theresa Wittman or PI Claire Singletary at 713-500-5599.

If you would like to be contacted to participate after you receive your NIPT results, please provide the following information:

Name:

Email: Phone Number:

Signature: Date:

If you agree to take part in the study, your agreement is completion of the survey. 
This research project has been reviewed by the Committee for the Protection of Human

Subjects (CPHS) of the University of Texas Health Science Center at Houston (HSC-MS-

15-0444). For any questions about research subjects rights, call CPHS at (713) 500-7943.

For genetic counselor use only:

Date:

Indication: AMA Positive FTS Screen Positive Quad Screen Ultrasound Abnormality Positive Family History

Other

Age:

G: P: 


\section{Appendix B}

\section{Prenatal Patient Questionnaire}

You were seen for genetic counseling during your current pregnancy and offered to participate in a survey about having a blood test called noninvasive prenatal testing, or NIPT. Thank you for agreeing to be contacted to discuss your feelings about NIPT.

This survey should take approximately 10 minutes to complete. Whether or not you choose to participate will not impact your care in any way. Your participation is greatly appreciated.

Please choose the best answer for each question:

1. How many years of education you have completed?
a. Never attended high school
b. Some high school
c. High school/GED
d. Some college or 2 year/associates degree
e. 4-year degree (BA, BS)
f. Graduate or Professional degree (MS, MBA, PhD, MD, JD)
g. Do not wish to answer 
2. What is your marital status?
a. Married/living with partner
b. Unmarried, living with other adults
c. Unmarried, living without other adults
d. Do not wish to answer

3. With which race/ethnicity do you most identify?
a. White, non-Hispanic
b. Hispanic
c. African American
d. Asian/Pacific Islander
e. Native American
f. Other:
g. Do not wish to answer

4. On a scale of 1 to 5 , with 1 being unconcerned and 5 being very concerned, please rate what your level of concern was for your baby to have any health problem before you had noninvasive prenatal testing (NIPT)

$$
\begin{array}{lllll}
1 & 2 & 3 & 4 & 5
\end{array}
$$

5. On a scale of 1 to 5 , with 1 being unconcerned and 5 being very concerned, please rate what your level of concern was to have a baby with a health problem, before you had noninvasive prenatal testing (NIPT)

$$
\begin{array}{lllll}
1 & 2 & 3 & 4 & 5
\end{array}
$$


6. You had a negative NIPT test. What do you feel that your chances are to have a baby with Down syndrome after receiving this result? After receiving a negative NIPT result I feel my chances are...

a. Not lower than before

b. Somewhat lower than before

c. Much lower than before

d. There is no longer a chance that my baby will have Down syndrome

7. On a scale of 1 to 5 , with 1 being unconcerned and 5 being very concerned, please select what your present level of concern is to have a baby with Down syndrome after a negative NIPT result

$$
\begin{array}{lllll}
1 & 2 & 3 & 4 & 5
\end{array}
$$

8. What are your chances to have a baby with trisomy 18 or trisomy 13 after receiving a negative NIPT result? After receiving a negative NIPT result I feel my chances are...

a. Not lower than before

b. Somewhat lower than before

c. Much lower than before

d. There is no longer a chance that my baby will have trisomy 13 or trisomy 18

9. On a scale of 1 to 5 , with 1 being unconcerned and 5 being very concerned, please select what your present level of concern is to have a baby with a baby with trisomy 18 or trisomy 13 after receiving a negative NIPT result

$$
\begin{array}{lllll}
1 & 2 & 3 & 4 & 5
\end{array}
$$


10. What are your chances to have a baby with a sex chromosome condition such as Turner syndrome or Klinefleter syndrome after receiving a normal NIPT result?

After receiving a negative NIPT result I feel my chances are...
a. Not lower than before
b. Somewhat lower than before
c. Much lower than before
d. There is no longer a chance that my baby will have a sex chromosome condition

11. On a scale of 1 to 5 , with 1 being unconcerned and 5 being very concerned, please select what your present level of concern is to have a baby with a sex chromosome condition after a negative NIPT result.

$$
\begin{array}{lllll}
1 & 2 & 3 & 4 & 5
\end{array}
$$

12. What are your chances to have a baby with a genetic condition other than Down syndrome, trisomy 18 , trisomy 13 or a sex chromosome conditions after receiving a normal NIPT result? After receiving a negative NIPT result I feel my chances are...
a. Not lower than before
b. Somewhat lower than before
c. Much lower than before
d. There is no longer a chance that my baby will have any genetic condition other than other than Down syndrome, trisomy 18 , trisomy 13 or a sex chromosome
condition

13. Please select your level of worry about having a baby with a genetic condition other than Down syndrome, trisomy 18 , trisomy 13 or sex chromosome condition

$$
\begin{array}{lllll}
1 & 2 & 3 & 4 & 5
\end{array}
$$


14. Please select whether NIPT is able to specifically test for any of the following:
a. Spina bifida
Yes
No
Don't know
b. Cleft lip
Yes
No
Don't know
c. Gender
Yes
No
Don't know
d. Structure of heart
Yes
No
Don't know
e. Intellectual disability
Yes
No
Don't know
f. Autism
Yes
No
Don't know
g. Diabetes
Yes
No
Don't know

15. Have you had your anatomy ultrasound (typically performed around 20 weeks of pregnancy)? $\quad$ Yes No

If yes, were any abnormalities found on your ultrasound?

If yes, please describe:

16. Did you have an amniocentesis procedure (needle test) or chorionic villus sampling (CVS) after receiving your negative NIPT results? $\quad$ Yes No

17. Are you planning to have an amniocentesis procedure (needle test) or chorionic villus sampling (CVS)? after receiving your negative NIPT results? Yes No

18. If yes, why did you or why will you have an amniocentesis or CVS? (check all that apply)

- Concerns from my NIPT

- Concerns on an ultrasound

- For piece of mind

- For greater accuracy

- To test for other conditions

- Other: 
19. If you did not or will not have an amniocentesis (needle test) or chorionic villus sampling (CVS), why not? (check all that apply)

- NIPT results were reassuring enough

- My doctor/genetic counselor said I did not need it

- The risk of miscarriage

- I am not worried about other conditions NIPT did not test for

- I am not worried about Down syndrome, trisomy 18 , trisomy 13 or sex chromosome conditions

- I do not like needles

- I will continue the pregnancy regardless of a genetic condition

- Other:

20. If your NIPT testing had come back abnormal, were you planning to have an amniocentesis or CVS?

$$
\text { Yes No Unsure }
$$

Why or Why Not?

21. How has having a negative NIPT test impacted your worry level about the pregnancy? (please check)
a. I worry much less
b. I worry a little less
c. I worry about the same
d. I worry a little more
e. I worry a great deal more 
e406 Patient Perception of Negative Noninvasive Prenatal Testing Results Wittman et al.

22. Rank from most important (1) to least important (6) the reasons behind your decision to pursue NIPT?

To determine my baby's gender

To determine my baby's chance of having Down syndrome

To determine my baby's chance of having another chromosome condition, such as

trisomy 18 , trisomy 13 , or a sex chromosome disorder

To avoid having amniocentesis or CVS

To make my doctor happy

To relieve anxiety

23. Please describe below any other reason not listed above that was important in your decision- to pursue NIPT

24. Please describe below any other comments or concerns you have regarding your noninvasive prenatal testing? 